\title{
Concerns of patients with systemic lupus erythematosus and adherence to therapy - a qualitative study
}

This article was published in the following Dove Press journal:

Patient Preference and Adherence

14 July 2017

Number of times this article has been viewed

Filipa Farinha'

Francisco Freitas ${ }^{2}$

Ana Águeda'

Inês Cunha'

Anabela Barcelos'

'Rheumatology Department, Centro Hospitalar do Baixo Vouga EPE, Aveiro, Portugal; ${ }^{2}$ Centre for Social Studies, Universidade de Coimbra, Coimbra, Portugal
Correspondence: Filipa Farinha Serviço de Reumatologia - Centro Hospitalar do Baixo Vouga, Av. Artur Ravara, 38I0-50I Aveiro, Portugal

Tel +35I 234378300

Fax +35I 234378395

Email filipa.mcfarinha@gmail.com
Purpose: The objectives of this study were 1) to identify the impact of systemic lupus erythematosus (SLE) on patients' lives and their reactions to this, as well as their main concerns and expectations regarding their disease and treatments; and 2) to assess the relationship between these concerns and the adherence to treatments, medical visits, and diagnostic tests.

Patients and methods: Qualitative study, using a convenient sample of SLE patients attending an outpatient rheumatology clinic. Semistructured interviews were conducted and audiotaped. The full transcripts were analyzed by two different coders using content analysis methodology.

Results: Fifteen participants were included. SLE had a major impact on these patients' lives. Their main concerns were fear of disease worsening and becoming dependent on other people, fear of not being able to take care of their children or provide for the family, and the possibility of transmitting SLE to their offspring. The main reasons for adherence to therapy were the wish to avoid manifestations of SLE and trust in the rheumatologist and routine. Nonadherence was more common in the beginning of the treatment because of the difficulty in accepting a chronic disease that requires lifelong therapy.

Conclusion: Our data underlined the important interplay between adherence to medication and the possibility to gather accurate information and proper support during the treatment process. Good communication and efficient patient education strategies, focused on improving their knowledge about the disease and its treatments, may be important to improve adherence to therapy in SLE.

Keywords: illness perception, adherence, communication, patients' perspectives, qualitative research

\section{Introduction}

Systemic lupus erythematosus (SLE) is a complex chronic autoimmune rheumatic disease with a marked female predominance. It can affect multiple organ systems and is responsible for increased morbidity and mortality.

There is evidence that adequate treatment can improve clinical outcomes. ${ }^{2}$ However, studies have shown high rates of nonadherence in SLE patients. ${ }^{3}$ Some studies have focused on the reasons for noncompliance with pharmacological therapy. ${ }^{4,5}$ The explanations found in a qualitative study with 31 interviews were "the belief that lupus could and should be controlled using alternative methods, the belief that long-term use of drugs was not necessary, the fear of drug adverse effects, practical difficulties in obtaining medications, and poor communication between patients and physicians". ${ }^{4}$ In Portugal, as opposed to the UK and Jamaica, where Chambers and collaborators 
conducted their research, ${ }^{4,5}$ the national healthcare system provides SLE patients all their prescribed medications, free of charge, regardless of whether they are followed in a public hospital or a private practice. Therefore, we presume Portuguese patients will differ from those in the UK and Jamaica in terms of reasons for nonadherence.

Illness perception has an important influence on treatment adherence and disease outcomes. ${ }^{6,7}$ Some studies explored factors influencing SLE patients' perception of their illness and its treatment. A recent quantitative study ${ }^{6}$ suggested a significant relationship between alexithymia and several aspects of SLE patients' perceived health. This relationship seemed to be mediated by the development of depressive symptoms, which played an important role in "modulating patients' perception of treatment efficacy and emotional responses to illness". ${ }^{6}$ Other studies supported the association of illness perceptions with levels of depression. ${ }^{8}$

Qualitative approaches can provide richer information about patients' perspectives and their relationships with healthcare providers. ${ }^{9}$ This kind of studies has been widely used in musculoskeletal research, ${ }^{10}$ in some cases including the use of computer-assisted qualitative data analysis software. ${ }^{11,12}$ However, few studies have addressed the patient's perspective of how SLE affects their lives. We believe that the concerns and expectations of SLE patients influence their adherence to treatments and also their compliance with medical visits and diagnostic tests proposed. Therefore, our objectives were 1) to identify the impact of SLE in patients' lives and their reactions to this, as well as their main concerns and expectations regarding their disease and treatments and 2) to assess the relation between these concerns and the adherence to treatments, medical visits, and diagnostic tests.

\section{Patients and methods}

We performed a qualitative content analysis study using a convenient sample of SLE patients attending an outpatient rheumatology clinic in a secondary care center. Patients who had a medical visit or diagnostic tests scheduled for the dates stipulated for the interviews to take place, were invited, via telephone, to meet the principal investigator (FF) on the same day they would be coming to the hospital (to avoid extra dislocations). Patients who accepted the invitation were explained face-to-face, by the main researcher, the objectives and design of the study. To be included in the study, patients had to meet the following criteria: age $\geq 18$ years old; disease duration $\geq 1$ year; fulfillment of American College of Rheumatology (ACR) ${ }^{13}$ or Systemic Lupus International Collaborating Clinics (SLICC) ${ }^{14}$ SLE classification criteria; and willingness to participate and able to give informed signed consent. Patients with cognitive impairment were excluded.

Qualitative studies normally address a limited number of cases and include small pools of unstructured rich data. Sampling units are purposively generated to achieve data saturation. Saturation or redundancy is defined as the point when no new information is obtained from further data. This is a strategy to determine a sample size in qualitative research that indicates the adequate amount of cases to be considered. For this type of study, there are no fixed amount of cases or standard tests to determine the required data to reach saturation, and data collection is very often performed alongside the data analysis. ${ }^{15}$

Semistructured face-to-face interviews were conducted by $\mathrm{FF}^{1}$ in a quiet room, in the hospital. This researcher was a female Specialist Registrar in Rheumatology with a special interest in SLE, but with no previous relationship with the participants. Interviews, with an approximate duration of 30-40 minutes, were conducted in Portuguese, the native idiom of both the participants and the interviewer, and audiotaped in an anonymized manner. The topics approached included demographic data, disease duration and evolution, treatments, rheumatology medical visits, adherence, meaning of the disease and its implications, main concerns, and expectations and communication with the rheumatologist. One pilot interview (not included in the analysis) was performed to test and adjust the protocol.

The full transcripts were integrated into a MAXQDA (VERBI GmbH, Germany) project file. The data were analyzed by two different coders $\left(\mathrm{FF}^{1}\right.$ and $\mathrm{FF}^{2}$ ) using content analysis methodology ${ }^{16,17}$ to identify the emerging themes and to code the data exhaustively. A codebook was discussed and implemented (Table 1) in the software. The codebook was refined during the data analysis process. The refinement was conducted using the Intercoder Agreement function available in MAXQDA, variation 3 (ie, measurement of agreement/ alignment of each individual coded segment). The Intercoder Agreement function allowed the comparison and contrast of single documents coded by the two coders. For this study, the agreement of each individual coded segment was thus measured for the first five interviews considering a $90 \%$ agreement percentage. The aim was to decide which coding variation in each situation was most accurate, in order to improve the codebook (ie, to clarify the definition of the subcodes) and to inductively generate new entries in the codebook to cover absent themes.

The study was conducted in agreement with the Declaration of Helsinki and was approved by the local ethics 
Table I Analysis grid (MAXQDA codebook)

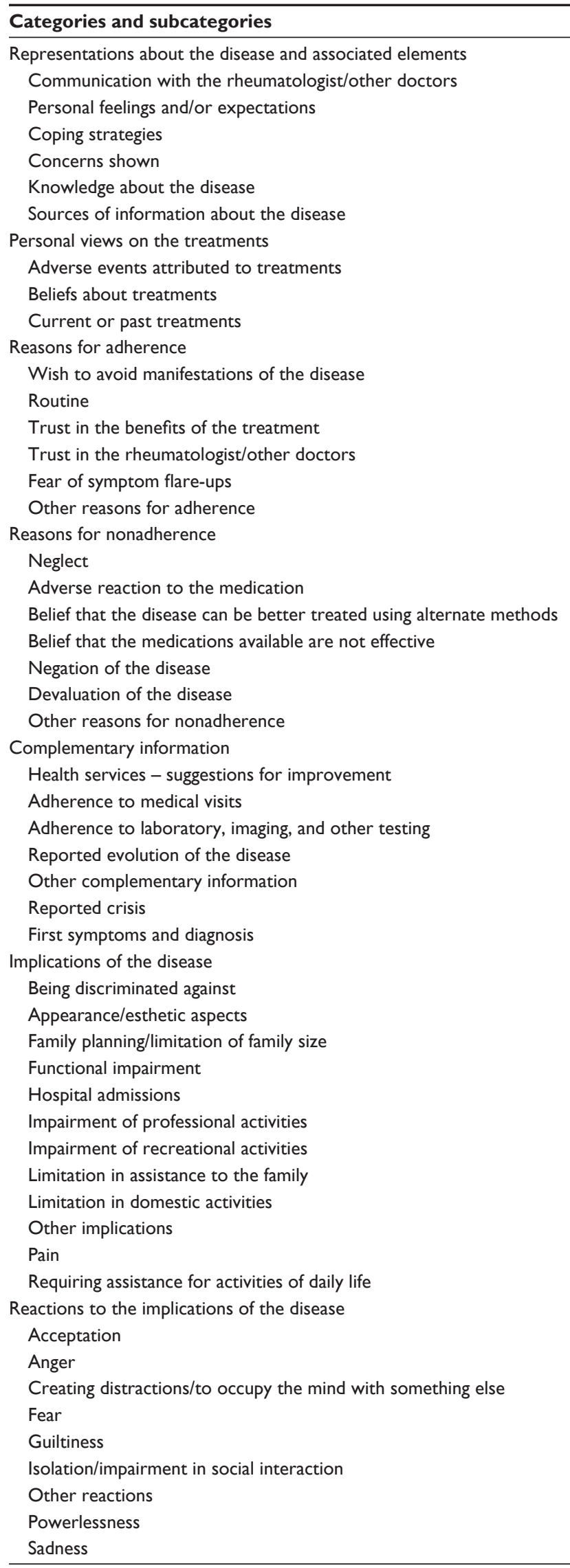

committee (Comissão de ética do Centro Hospitalar do Baixo Vouga, EPE; reference: 15FEB'16 12:03 067770).

\section{Results}

Sixteen patients were interviewed and 15 were included in the analysis, 14 females and one male (the pilot interview was not included). The participants were all Caucasians and had a median age of 40 (interquartile range [IQR]: 36-45.5) years and a median disease duration of 12 (IQR: 7-16) years. Their characteristics are shown in Table 2. Five other patients were invited to meet the main researcher: four of them argued that they did not have time; one accepted the invitation but missed the appointment. All the patients who met the investigator

Table 2 Characteristics of the participants

\begin{tabular}{|c|c|}
\hline$\overline{\mathbf{N}}$ & 15 \\
\hline Age, median (range), years & $40(23-60)$ \\
\hline \multicolumn{2}{|l|}{ Gender } \\
\hline Female & 14 \\
\hline Male & I \\
\hline \multicolumn{2}{|l|}{ Education, year } \\
\hline$\leq 6$ & 2 \\
\hline $7-12$ & 3 \\
\hline Higher & 8 \\
\hline \multicolumn{2}{|l|}{ Employment } \\
\hline Full time & 10 \\
\hline Unemployed & 1 \\
\hline Housewife & 4 \\
\hline \multicolumn{2}{|l|}{ Marital status } \\
\hline Married/civil union & 14 \\
\hline Single & I \\
\hline Duration of SLE, median (range), years & $12(2-23)$ \\
\hline \multicolumn{2}{|l|}{ Manifestations } \\
\hline Immunological & 15 \\
\hline Mucocutaneous & 12 \\
\hline Hematological & 8 \\
\hline Articular & 8 \\
\hline Renal & 5 \\
\hline Serositis & I \\
\hline \multicolumn{2}{|l|}{ Immunosuppressants } \\
\hline Hydroxychloroquine & 14 \\
\hline Corticosteroids & 13 \\
\hline Azathioprine & 3 \\
\hline Methotrexate & 1 \\
\hline Mycophenolate mofetil & I \\
\hline Cyclosporin & I \\
\hline \multicolumn{2}{|l|}{ Other medications } \\
\hline ACEl or ARB & 7 \\
\hline Bisphosphonates & 2 \\
\hline Calcium & 7 \\
\hline Warfarin & I \\
\hline Low-dose aspirin & 6 \\
\hline NSAIDs & I \\
\hline
\end{tabular}

Abbreviations: ACEl, angiotensin-converting enzyme inhibitor; ARB, angiotensin II receptor blocker; NSAIDs, nonsteroidal anti-inflammatory drugs; SLE, systemic lupus erythematosus. 
and were explained about the study agreed to participate. The data obtained from this first round of 15 interviews granted a correct level of theoretical saturation. Therefore, it was not necessary to add entries to the initial pool of data since the research topics were fully covered and emerging themes inserted in the codebook accordingly.

The participants reported different experiences regarding their disease. Some of them had relatively mild manifestations; for others, SLE was a very debilitating disease. For all of them, though, Lupus was a constant presence.

\section{Impact of SLE and reactions to this}

This group of patients found several limitations in their lives as a consequence of the disease. First of all, they reported pain, fatigue, and other symptoms that interfered with dailylife, domestic, and professional activities, and with their social and sexual lives.

Participant 1: My husband had to bathe me because I couldn't; I want to do a good cleaning in my house but many times my hands don't let me do so.

Participant 5: I used to sell fish in the market. I had to quit because of ... my hands.

Participant 9: The fact that I feel very sleepy, very tired [...] interferes with life with my husband because sometimes libido goes away; I have a very good group of friends. [...] We used to go out but sometimes I can't. Then if, for instance, we meet at my place or theirs [...] they laugh, because I fall asleep.

Participant 15: Having a 5-month-old child and not being able to breastfeed him. [...] I could not hold him. I could not bathe him ... Anything ... Because I was lying in bed. It was very hard.

Another implication perceived by the participants was the need to avoid exposure to the sun and, therefore, to some outdoor leisure activities. These patients lived at the seaside and most of them wished they could go to the beach as they did before they had the disease.

Participant 4: For instance, imagine meeting up people [...] it [SLE] limits [...] even a simple picnic in a summer day.

Participant 14: Not being able to go to the beach.

It leaves me very sad.

Esthetic issues associated with SLE were also reported. In fact, appearance changes related to rashes, alopecia, and visible joint swelling negatively affected these patients' lives.

Participant 5: I couldn't look at the mirror and I was upset

by people staring at me. Because in summer I am very red and in winter I get completely blue. And people stop [...]

in the supermarket $[\ldots]$ and stare.

Reactions to their disease evolved with time. From the analysis of the interviews, we could realize that this was a process with several stages. Initially, there was the difficulty in coping with the diagnosis of a chronic disease with all its implications. Most participants reported some kind of psychological effect and some of them needed help from psychiatrists or psychologists.

Participant 1: But I had moments when I felt very angry,

I got to the point of hurting myself [...] I did a cut here, I hit

the window with my head. Hum, angry because by that time

we were planning to buy a house and all that affected me.

This group of participants did not show resignation; on the contrary, they tried to fight the disease and/or to minimize its consequences. At some point, they went through a process of adaptation of their lives to the new reality and used several strategies to cope with the disease, most of which consisted of keeping an active life and trying not to think too much about the disease and its consequences. In the end, all of them accepted this condition with an attitude of normalization of its impact. In fact, most said they had a normal life, even though it is possible to infer, from the analysis of the interviews, that multiple aspects severely conditioned their lives (restrictions related to the disease, chronic medication, pain, etc.).

\section{Concerns}

Participants were asked about their main concerns regarding their disease and treatments. Emergent themes about this topic were mainly the fear of disease worsening and, consequently, losing autonomy and becoming dependent on others. They also feared that they would not be able to take care of their children or to provide for the family, and feared the possibility of transmitting SLE to their offspring.

Participant 5: [I worry about] ending up in a wheelchair and becoming a burden [to my family].

Participant 1: I could only think, what if I become paralyzed and cannot take care of the house, and mainly my daughter [...] who was still a small child.

Participant 3: I wonder whether I'm being an irresponsible person, for having three children, knowing they may have my disease.

Although all participants of our study stated that their rheumatologists were attentive and always answered their questions, some of them still had doubts about the disease, namely, the possible consequences of it, which 
created anxiety and other negative feelings. These patients admitted that they did not usually share their concerns during medical visits, either because they forgot about it or because they did not feel it was opportune, as participant 3 said, "That moment is not always provided, you know? Because appointments are one after the other and $[. .$.$] you$ [the doctors] give us enough information but do not stay there ... talking, right?"

\section{Adherence}

The wish to avoid manifestations of SLE was the main reason for adherence to therapy. In fact, several patients realized that they felt worse if they stopped the medication. For instance, participant 5 said, "I have to take them [the medicines] in the morning otherwise I'm not able to walk." Therefore, medication appears here as a negative reinforcer, since patients adhere mostly to avoid pain and other symptoms. Most members of the group were able to express a very good level of understanding regarding the therapy, namely, the side effects related to some of the medicines.

Another important emerged reason for adherence was the trust in the rheumatologist. This became particularly clear with participant 10's interview: "I think it's more important [...] to trust in the person [the doctor] that is seeing us [...] in that case they can prescribe 50 tablets and we take them."

Most patients mentioned that they integrated medication in their daily routine, as participant 10 said, "It's like having my yogurt in the morning. It doesn't bother me, because it is a habit."

These patients felt more confident/reassured knowing that they had periodic clinical and laboratory evaluation. This was the main reason for them to attend the visits and to accept laboratory and other proposed tests.

Participant 9: Therefore, as I do them [the laboratory tests] every 4 months, I think $[\ldots]$ that reassures me $[\ldots]$ a lot.

Nonadherence was not common in this sample. It was reported in the beginning of the treatment because of the difficulty in accepting a chronic disease that requires lifelong therapy. Other reasons for nonadherence included oblivion, neglect, and adverse effects of the medication.

Participant 4: But in the beginning of course it was hard to realize that from that day on I had to take medicines and it's still hard when I'm told: You have to take also this, for all your life. It is more medication. [...] The day gets sad.

Participant 9: I used to stop medication from May or June on. It was to get slimmer to go to the beach.

\section{Discussion}

The impact of SLE in these patients' lives was mainly characterized by pain, fatigue, and other symptoms that interfered with daily-life, domestic, and professional activities, and with their social and sexual lives; having to avoid outdoor leisure activities; and esthetic issues. These patients went through a process of acceptation and adaptation of their lives because of the disease. Their main concerns included fear of disease worsening and becoming dependent, fear of not being able to take care of their children or to provide for the family, and the possibility of transmitting SLE to their offspring. The main reasons for adherence to therapy were the wish to avoid manifestations of SLE and trust in the rheumatologist and routine. These patients felt reassured knowing that they had periodic clinical and laboratory evaluation. Nonadherence was more common in the beginning of the treatment because of the difficulty in accepting a chronic disease that requires lifelong therapy. The other reasons reported were oblivion, neglect, and adverse effects of the medication.

Regarding the impact of lupus in patients' lives, other studies have shown similar results. ${ }^{18,19}$ Household responsibilities, parenting roles, recreational activities, work performance, and scholastic achievement have been reported to be affected by the signs and symptoms of SLE. ${ }^{18}$

A recent study investigated the interactions between body image, self-image, medication use, and adherence to medication in SLE patients. As in our study, patients revealed worries about appearance and weight, which they often related with the use of steroids, and also described "creative non-compliance". In that study, participants "felt their care providers did not give enough consideration to their concerns" about the effects of lupus and its treatment on the appearance. ${ }^{20}$ In contrast, although participants in our study appeared to be happy with the relationship they had with their rheumatologists, many of them tended not to share their concerns.

Another study, which assessed patients with one or more chronic diseases, suggested that there is a significant relationship between knowledge about medications and the tendency to adhere to long-term treatments. ${ }^{21}$ The same was suggested by a study in patients with rheumatoid arthritis: patients who reported lower adherence were more dissatisfied with the information they had received about their diseasemodifying antirheumatic drugs. ${ }^{22}$ Rao et al found that the most common unfulfilled expectation among patients with rheumatic diseases during their visits to rheumatologists was that for information. ${ }^{23}$ From our data, it was possible to 
infer that lack of information strongly relates to feelings of uncertainty and fear.

In summary, good communication between physician and patient appears to be essential to improve adherence. It may require proper adjustment to patients' cultural background as they describe disease and its effects in different ways. As shown in previous studies, it is important to involve the patients in decision-making and to help them feel comfortable and confident in sharing their questions and concerns. ${ }^{21}$ This will also enable a trustful relationship, which is, according to our findings, an important reason for adherence.

Our group of patients tended to adhere well and one of the main reasons for this seemed to be the direct link that they found between taking the medication and controlling the symptoms related to the disease. Therefore, compliance with treatments is rewarded with the possibility of living a normal life (although it is possible to understand that the very concept of normality for Lupus patients comprises a considerable list of adaptation strategies). However, since participants were recruited among patients who went to the hospital to have a medical visit or a laboratory test, it was possible that only compliant patients were invited and agreed to participate. If this was the case, our sample might not allow enough variability for a thorough assessment of motives influencing SLE patients' adherence to treatment. On the other hand, it may have happened that some patients did not disclose nonadherence, as they would have felt uncomfortable admitting it in a face-to-face interview. The absence of an anonymous quantitative questionnaire to assess treatment adherence was, therefore, a limitation of our study, as adherence was only assessed through the interviews. It is important to note that in Portugal, patients with SLE do not have to pay for their prescribed medications; therefore, in contrast to other populations, the cost was not the reason for poor adherence to pharmacological treatments.

Our study had a limited group of participants, which is common in qualitative research and is not seen as a problem, since theoretical saturation was achieved. In addition, participants differed in terms of disease duration, severity of manifestations, and social status. This inhomogeneity is usually a characteristic of samples in qualitative studies, as it may provide richer information. In fact, these kinds of studies are not usually expected to produce generalizable results as would be the case with quantitative research. ${ }^{20,24}$ Instead, they give us important information that allows us to understand this group of patients better and to generate concepts that can be transferable and used in clinical practice or in future studies. Nevertheless, in light of the Proximal Similarity Model, it could be licit to generalize the results of our study if there are strong "similarities between the time, place, people and other social contexts". ${ }^{24}$ An expansion of this study, to include a broader population with respect to adherence levels, would be important to understand better the reasons for nonadherence and to allow for the generalizability of the results.

\section{Conclusion}

Our research data underlined the important interplay between adherence to medication and the possibility to gather accurate information and proper support during the treatment process. The latter is particularly relevant considering the reported psychological effects of a disabling chronic disease requiring lifelong therapy. Good communication between physician and patient may enable trust and, therefore, improve adherence. In addition, efficient patient education strategies, focused on improving their knowledge about the disease and its treatments, may be important to improve adherence to therapy in SLE.

\section{Acknowledgment}

The abstract of this paper was presented at the 10th European Lupus Meeting, as an oral communication, with interim findings. The abstract was published in "Meeting Abstracts" in Clinical and Experimental Rheumatology 34 (4 Suppl 99):S47.

\section{Disclosure}

The authors report no conflicts of interest in this work.

\section{References}

1. Chambers SA, Allen E, Rahman A, Isenberg D. Damage and mortality in a group of British patients with systemic lupus erythematosus followed up for over 10 years. Rheumatology (Oxford). 2009;48(6):673-675.

2. Ruiz-Irastorza G, Ramos-Casals M, Brito-Zeron P, Khamashta MA. Clinical efficacy and side effects of antimalarials in systemic lupus erythematosus: a systematic review. Ann Rheum Dis. 2010;69(1): 20-28.

3. Costedoat-Chalumeau N, Pouchot J, Guettrot-Imbert G, et al. Adherence to treatment in systemic lupus erythematosus patients. Best Pract Res Clin Rheumatol. 2013;27(3):329-340.

4. Chambers SA, Raine R, Rahman A, Isenberg D. Why do patients with systemic lupus erythematosus take or fail to take their prescribed medications? A qualitative study in a UK cohort. Rheumatology (Oxford). 2009;48(3):266-271.

5. Chambers S, Raine R, Rahman A, Hagley K, De Ceulaer K, Isenberg D. Factors influencing adherence to medications in a group of patients with systemic lupus erythematosus in Jamaica. Lupus. 2008;17(8):761-769.

6. Barbasio C, Vagelli R, Marengo D, et al. Illness perception in systemic lupus erythematosus patients: the roles of alexithymia and depression. Compr Psychiatry. 2015;63:88-95.

7. Kotsis K, Voulgari PV, Tsifetaki N, Drosos AA, Carvalho AF, Hyphantis T. Illness perceptions and psychological distress associated with physical health-related quality of life in primary Sjogren's syndrome compared to systemic lupus erythematosus and rheumatoid arthritis. Rheumatol Int. 2014;34(12):1671-1681. 
8. Philip EJ, Lindner H, Lederman L. Relationship of illness perceptions with depression among individuals diagnosed with lupus. Depress Anxiety. 2009;26(6):575-582.

9. Paskins Z, Hassell AB. Qualitative research in RA. Rheumatology. 2012;51(1):3-4.

10. Ong BN, Richardson JC. The contribution of qualitative approaches to musculoskeletal research. Rheumatology (Oxford). 2006;45(4): 369-370.

11. Hayden C, Neame R, Tarrant C. Patients' adherence-related beliefs about methotrexate: a qualitative study of the role of written patient information. BMJ Open. 2015;5(5):e006918.

12. Moverley AR, Vinall-Collier KA, Helliwell PS. It's not just the joints, it's the whole thing: qualitative analysis of patients' experience of flare in psoriatic arthritis. Rheumatology (Oxford). 2015;54(8):1448-1453.

13. Hochberg MC. Updating the American College of Rheumatology revised criteria for the classification of systemic lupus erythematosus. Arthritis Rheum. 1997;40(9):1725.

14. Petri M, Orbai AM, Alarcon GS, et al. Derivation and validation of the Systemic Lupus International Collaborating Clinics classification criteria for systemic lupus erythematosus. Arthritis Rheum. 2012; 64(8):2677-2686.

15. Silverman D, Marvasti A. Doing Qualitative Research: A Comprehensive Guide. SAGE Publications; 2008.

16. Krippendorff K. Content Analysis: An Introduction to Its Methodology. 3rd ed. California: SAGE Publications; 2013.
17. Neuendorf K. The Content Analysis Guidebook. California: SAGE Publications; 2002.

18. Robinson D Jr, Aguilar D, Schoenwetter M, et al. Impact of systemic lupus erythematosus on health, family, and work: the patient perspective. Arthritis Care Res (Hoboken). 2010;62(2):266-273.

19. McElhone K, Abbott J, Shelmerdine J, et al. Development and validation of a disease-specific health-related quality of life measure, the LupusQol, for adults with systemic lupus erythematosus. Arthritis Rheum. 2007;57(6):972-979.

20. Hale ED, Radvanski DC, Hassett AL. The man-in-the-moon face: a qualitative study of body image, self-image and medication use in systemic lupus erythematosus. Rheumatology (Oxford). 2015;54(7): $1220-1225$.

21. Awwad O, Akour A, Al-Muhaissen S, Morisky D. The influence of patients' knowledge on adherence to their chronic medications: a crosssectional study in Jordan. Int J Clin Pharm. 2015;37(3):504-510.

22. Kumar K, Raza K, Nightingale P, et al. Determinants of adherence to disease modifying anti-rheumatic drugs in White British and South Asian patients with rheumatoid arthritis: a cross sectional study. BMC Musculoskelet Disord. 2015;16:396.

23. Rao JK, Weinberger M, Anderson LA, Kroenke K. Predicting reports of unmet expectations among rheumatology patients. Arthritis Rheum 2004;51(2):215-221.

24. Leung L. Validity, reliability, and generalizability in qualitative research. J Family Med Prim Care. 2015;4(3):324-327.
Patient Preference and Adherence

\section{Publish your work in this journal}

Patient Preference and Adherence is an international, peer-reviewed, open access journal that focuses on the growing importance of patient preference and adherence throughout the therapeutic continuum. Patient satisfaction, acceptability, quality of life, compliance, persistence and their role in developing new therapeutic modalities and compounds to optimize

\section{Dovepress}

clinical outcomes for existing disease states are major areas of interest for the journal. This journal has been accepted for indexing on PubMed Central. The manuscript management system is completely online and includes a very quick and fair peer-review system, which is all easy to use. Visit http://www. dovepress.com/testimonials.php to read real quotes from published authors. 